\title{
A mental picture of the greenhouse effect
}

\section{A pedagogic explanation}

\author{
Rasmus E. Benestad ${ }^{1}$
}

Received: 28 January 2015 / Accepted: 5 January 2016/Published online: 21 January 2016

(C) The Author(s) 2016. This article is published with open access at Springerlink.com

\begin{abstract}
The popular picture of the greenhouse effect emphasises the radiation transfer but fails to explain the observed climate change. An old conceptual model for the greenhouse effect is revisited and presented as a useful resource in climate change communication. It is validated against state-of-the-art data, and nontraditional diagnostics show a physically consistent picture. The earth's climate is constrained by well-known and elementary physical principles, such as energy balance, flow, and conservation. Greenhouse gases affect the atmospheric optical depth for infrared radiation, and increased opacity implies higher altitude from which earth's equivalent bulk heat loss takes place. Such an increase is seen in the reanalyses, and the outgoing longwave radiation has become more diffuse over time, consistent with an increased influence of greenhouse gases on the vertical energy flow from the surface to the top of the atmosphere. The reanalyses further imply increases in the overturning in the troposphere, consistent with a constant and continuous vertical energy flow. The increased overturning can explain a slowdown in the global warming, and the association between these aspects can be interpreted as an entanglement between the greenhouse effect and the hydrological cycle, where reduced energy transfer associated
\end{abstract}

Electronic supplementary material The online version of this article (doi:10.1007/s00704-016-1732-y) contains supplementary material, which is available to authorized users.

Rasmus E. Benestad

rasmus.benestad@met.no

1 The Norwegian Meteorological Institute, P.O. box 43 Blindern, 0313 Oslo, Norway with increased opacity is compensated by tropospheric overturning activity.

\section{Motivation}

It is usually an advantage knowing why your explanation makes sense when you try to make an impressions on others. The same can be said for explaining climate change to the general public and informing them about how the greenhouse effect (GHE) is altered by the burning of fossil fuels. Despite a strong consensus on climate change within the climate research community (Anderegg et al. 2010; Oreskes 2004; Cook et al. 2013), the efforts to explain climate change to the society so far can be summed up as having had limited success (Doran and Zimmerman 2011; Theissen 2011; Leviston et al. 2012; Lewandowsky et al. 2013; Rahmstorf 2012; Boykoff 2008; McCuin et al. 2014), and the greenhouse gas (GHG) concentrations have continued to rise (IPCC synthesis report A 2014). It is possible that the message from scientific organisations such as the Intergovernmental panel on Climate Change (IPCC) has been too cryptic to the layman (Benestad 2014b). On the other hand, it is unlikely that an increased appreciation for the scientific facts will prompt a change in habits (Hernes 2012). An improved understanding of the relevant science will nevertheless be helpful in the outreach efforts made by institutions such as national meteorological services. One of the greatest challenges has been that climate change and the GHE are complicated topics, and there has been a lack of convincing simplified description that people can conceptualise. The part of the society who has been educated in atmospheric sciences will probably already understand many features of the GHE; however, they too may benefit from a new angle in terms of explaining the concepts to 
others. The popular 'blanket model' is too unrealistic for the more scientific literate part of the population, hence, there is perhaps a need to provide a picture of how different climatic aspects depend on relevant boundary conditions that is convincing in terms of physics. If the science educated part of the society understands the reasons for climate change, then they may make a stronger impression on the general society. This could perhaps be achieved with a more pedagogic and more comprehensible description of anthropogenic global warming (AGW).

The essential principles of the GHE have long been understood (Weart 2003) in terms of simple reductionist models (Fleagle and Businger 1980; Houghton 1991; Peixoto and Oort 1992; Hartmann 1994; Lacis et al. 2010; Pierrehumbert 2011), however, these models usually don't provide a comprehensive description of the complexity associated with a global warming. Often they give a picture of the radiative energy transfer only (Fleagle and Businger 1980; Houghton 1991; Peixoto and Oort 1992; Hartmann 1994) demonstrating the essential principles behind the GHE, but they don't tell the complete story. For instance, Sloan and Wolfendale (2013) demonstrated how an elegant physics-based derivation leads to a simple equation describing the radiative fluxes involved in the GHE, explaining why the radiative forcing is proportional to $\ln (\rho)$, where $\rho$ is the density of GHGs in parts per volume. However, the solution for the radiation transfer alone is unrealistically sensitive to the GHG concentration, due to the omission of convective, sensible, and latent heat transfer. The reductionist radiative model explained the principle behind GHE, but failed to account for all relevant processes. A similar finding had already been derived by Manabe and Möller (1961).

Scholars within the science community may also have somewhat different ideas about what is involved in the GHE. Here, the GHE will, for all intents and purposes, be defined as the set of conditions that are responsible for discrepancy between the observed global mean surface temperature of a planet and that predicted based on the energy flux received from the sun, rather than being restricted to a mere radiative balance. The term 'model' too can have many different interpretations, and here it refers to a synthesis of a set of pieces of relevant knowledge that describe the essence of a phenomenon or a condition under investigation. All models are 'flawed' since they represent an idealistic description (neglecting aspects and details that are not of interest), however, they can still give valuable insights and useful predictions. A model may be as simple as an equation describing the incoming and outgoing energy flow, where the theoretical calculations are based on the conservation of energy. Such a model is presented here, based on the fact that the solar radiation absorbed by the planet must be balanced by the outgoing thermal radiation. These basic principles were first described by Joseph Fourier in 1824.
Present global climate models (GCMs) supersede the old simple conceptual models on the greenhouse effect, some of which include radiative-convection and heat balance models discussed over the period 1890-1980 (Arrhenius 1896; Hulburt 1931; Charney et al. 1979; Schneider and Dickinson 1974; North 1975; Wang WC and Stone P 1980). The stateof-the-art GCMs, however, are almost as complex as nature itself, making it hard to picture the connections between the most essential processes. Revisiting an old 'back-ofthe-envelope', proposed by Hulburt (1931), can provide us with an overview and an appreciation for how increased $\mathrm{CO}_{2}$-concentrations affect our atmosphere. The objective is to take a step back and re-examine our understanding of the GHE in light of new information in a similar spirit to Schneider and Dickinson, who provided some didactic value in addition to explaining the essence of the greenhouse effect (Schneider and Dickinson 1974).

The didactic aspect is relevant, as there is still a number of scientists who don't acknowledge the link between increased levels of greenhouse gas concentrations (GHGs) and climate change (Abraham et al. 2014; Anderegg et al. 2010) and dismiss the scientific literature supporting the notion of an AGW (Oreskes 2004; Solomon et al. 2007). There is a surprisingly high number of physicists among this group (Oreskes and Conway 2008), which suggests that old knowledge has been forgotten (Proctor and Schiebinger 2008). For instance, one paper published in International Journal of Modern Physics B (Gerlich and Tscheuschner 2009) claimed to falsify the greenhouse effect in its entirety (its claims, however, have subsequently been refuted (Halpern et al. 2010; Smith 2008)).

The objective here is to present Hulburt's 1931 model as a pedagogic description of the GHE, and test whether it is consistent with modern state-of-the-art data which were not available in 1931. The modern data include recent years as well as satellite-based measurements of outgoing long-wave radiation (OLR) and reanalyses. One major simplification involves treating all the infrared radiation (IR) as if it consisted of one wave length, although the real situation is of course much more complicated with a wave spectrum, selective absorption bands, and radiation with different frequencies and optical depths (Pierrehumbert 2011).

\subsection{A simplified physical picture}

The starting point for the conceptual model is the energy flow through the solar system, being generated inside the sun, intercepted by the planets, and flowing through the planetary system before escaping back to space (Trenberth and Stepaniak 2004). When the energy enters and leaves the terrestrial system, it must be in the form of electromagnetic radiation, since space is a vacuum and does not provide a 
medium for other types of energy transfer (Pierrehumbert 2011).

The radiative energy emitted from an object can be described by Planck's law, relating electromagnetic energy loss to a so-called 'emission temperature' $\left(T_{e}\right)$. The terrestrial energy loss is often referred to as the OLR, and the total energy loss may be approximated by a bulk flux (Hulburt 1931), although spectral lines due to atomic and molecular line absorption and quantum mechanics play an important role (Fleagle and Businger 1980; Houghton 1991; Pierrehumbert 2011). Here, 'bulk' is used in the meaning of being averaged over all altitudes and wavelengths. The rate of heat loss $\left(4 a^{2} \pi \sigma T_{e}^{4}\right)$ must equal the rate of energy received from the sun $\left(\pi a^{2} S_{0}(1-A)\right)$ for a planet (here $a$ is its radius) to be in energetic equilibrium (Hulburt 1931). The planetary energy balance can then be described approximately by the simple equation

$S_{0}(1-A) / 4=\sigma T_{e}^{4}$,

where $\sigma=5.67 \times 10^{-8} W /\left(m^{2} K^{4}\right)$ is the Stefan-Boltzmann constant, $A \sim 0.3$ is the albedo, and $S_{0}=1361 \mathrm{Wm}^{-2}$ is the 'solar constant' (Schneider and Dickinson 1974; Wang WC and Stone P 1980; Kopp and Lean 2011). The left hand side of this equation represents the energy input while the right hand side describes the bulk heat loss. Predicted temperatures according to Eq. 1 can be compared with actual observed surface temperatures in our solar system to demonstrate predictive skill (see Fig. S3 in the supporting material, hereafter referred to as Supplementary Material). Furthermore, the value of $T_{e}$ derived from measured terrestrial OLR is typically in the range 251-254 K (Fig. S4 in the Supplementary Material), providing a crude means for validating Eq. 1.

Equation 1 implies a value of $T_{e}=254 \mathrm{~K}$ for the terrestrial emission temperature, whereas the observed global mean surface temperature is $T \approx 288 \mathrm{~K}$. The bulk of the heat loss to space cannot take place at the ground level where $T=288 \mathrm{~K}$ because this would violate the energy balance (1). However, it has long been established that the bulk planetary heat loss is determined by atmospheric temperatures far above the surface (Hulburt 1931; North 1975). The temperature drops with height due to convective adjustment (standard atmosphere vertical temperature profile with decreasing temperature with height) and the radiative heating profile (Fleagle and Businger 1980; Houghton 1991; Peixoto and Oort 1992; Hartmann 1994), and equals the emission temperature of $254 \mathrm{~K}$ at around $6.5 \mathrm{~km}$ above the ground (Fig. S10 in the Supplementary Material). This altitude is where earth's bulk heat loss (i.e., the average based on all wave lengths and all altitudes) must take place according to Eq. 1. This is also independently corroborated by $T_{e}$ derived from measured OLR (see Fig. S4, Supplementary Material).

The basic principle of the GHE in general terms is that the air is opaque to light in the long-wave range (IR) but transparent to short-wave radiation (visible light). Most of the energy from the sun that is not reflected away is absorbed at the planet's surface, as a cloud-less atmosphere is transparent to visible light while clouds tend to reflect it (accounted for through the albedo $A$ ). On average, the net short-wave energy flux (visible light) on earth is roughly $240 \mathrm{~W} / \mathrm{m}^{2}$, which must balance an equal upward energy flow for a planet in equilibrium. The degree of transparency of a medium is described by Beer's law, which relates the optical depth to the density of the absorbing medium and its absorbing capacity (Fleagle and Businger 1980; Peixoto and Oort 1992). Several atmospheric gases (e.g., $\mathrm{H}_{2} \mathrm{O}, \mathrm{CO}_{2}$ in addition to clouds) are opaque in dominant frequency ranges predicted by Planck's law for $T \approx 288 \mathrm{~K}$. Indeed, the atmospheric $\mathrm{CO}_{2}$-concentrations are specified from IR gas analyzer measuring the amount of IR light absorbed in air samples (Keeling et al. 1976).

In the atmosphere, IR light can be absorbed and reemitted multiple times before its energy reaches the emission level where it is free to escape to space (Pierrehumbert 2011). The process of repeated absorption and re-emission will result in a more diffuse structure for the OLR at the top of the atmosphere. Hence, for an observer viewing the earth from above (e.g., a satellite instrument measuring the OLR), the bulk IR light source is expected to be both more diffuse and located at increasing heights with greater concentrations of GHGs, as the depth to which the observer can see into the atmosphere gets shallower for more opaque air. This altitude is henceforth referred to as the 'equivalent bulk emission level' and is the $254 \mathrm{~K}$ isotherm $Z_{T 254 K}$. It represents the mean height for both cloudy and cloud-free regions.

Higher concentrations of greenhouse gases divert more IR radiation downwards toward the ground (Trenberth 2011), as a deeper optical depth, due to increased absorption, is expected to restrict the radiative energy transfer, everything else being constant. Moreover, a change in the opacity will alter the radiative energy flow from the surface to the emission-level, which needs to be compensated by other forms of energy flow if the planet is to remain in energy balance according to Eq. 1 and if the total energy transfer is to be a continuous flow. In other words, the reduced radiative energy flux must be compensated through increased temperatures or altered latent/sensible heat fluxes.

The radiative equilibrium temperature gradient (Manabe and Strickler 1964) is also unstable in the troposphere (the temperature decreases with altitude), and convection sets in and takes over a significant amount of the vertical energy flow. Whereas the added opacity will act to restrict 
the flow of radiative energy transport, convection will not allow the temperature gradient to increase. In other words, a bigger share of the $240 \mathrm{~W} / \mathrm{m}^{2}$ of the vertical energy transport will be transported by convective/advective means with a stronger GHE, and a smaller share by radiative means because the sum of convective vertical energy transport plus the diminished radiative flux must add up to about 240 $W / m^{2}$ in order to balance the incoming shortwave radiation.

The above is an oversimplified picture, of course. Solar energy is absorbed throughout the atmosphere (e.g., suspended particles), not only at the ground. Non-radiative energy transports require a capable 3D general circulation model to render properly, and radiative transfer modeling must resolve the spectral dependence of all of the atmospheric radiative constituents. The basic concepts outlined above, however, should still be applicable in theory. These concept include a trend in the altitude of the emission level consistent with the global warming, a stronger interference between upwelling IR from the ground and absorbing matter and increased vertical overturning. This paper will examine the actual numbers and test the veracity of this conceptual description.

\section{Methods and data}

The conceptual picture of the GHE can be examined in terms of estimates of $Z_{T 254 K}$, the 'fuzziness' of the OLR, and a quantification of the vertical energy flow associated with other forms than radiative fluxes. These quantities are examined in the context of the constraints provided by the simple physics represented in Eq. 1 and the documented presence of a historic increase in the GHGs concentrations (Stocker et al. 2013).

The height of $Z_{T 254 K}$ was estimated from the European Centre for Medium-range Weather Forecasts (ECMWF) interim reanalysis (ERAINT) (Simmons et al. 2007) (37 model levels between 1000 and $1 \mathrm{hPa}$ which corresponds to $\sim 0 \mathrm{~m}$ and $\sim 63000 \mathrm{~m}$ above sea level). Monthly mean values for $T$ were estimated from instantaneous values, sampled at four times a day over the interval with available data January 1979-September 2012 at the full $0.75^{\circ} \times 0.75^{\circ}$ horizontal resolution.

Convection is characterized by a circulation pattern of rising and sinking air masses, and the atmospheric vertical volume transport takes place through cells of updraft and subsidence; however, these may not be coherent or stable in time and space. It was therefore important to find a measure that does not assume patterns that are stable in time (see Supplementary Material). Sensible and latent heat transfer are related to the vertical motion, but not completely determined by the flow, as the atmospheric vapor content and temperature gradients also are important factors. However, water vapor content is poorly constrained, due to uncertainties associated with clouds and precipitation. The vertical velocity $(w)$, on the other hand, is related to horizontal air flow through divergence, convergence, and variation in well-described quantities such the geopotential heights and barometric pressure. The monthly mean vertical velocity $w$ from ERAINT was used to provide a measure for the global atmospheric overturning by estimating the variance in $w$ over space for each time step separately (see Supplementary Material).

In addition to the ERAINT reanalysis, the NCEP/NCAR (Kalnay et al. 1996) and ERA40 (Simmons and Gibson 2000) reanalyses were also used as these covered longer time intervals. OLR measured from space (Liebmann 1996) was used to test equation 1 , derive $T_{e}$, and examine trends in diffuse IR emission at the top of the atmosphere. A spatial correlation was used as a measure of the degree of diffuse IR emission because it is insensitive to trends in the absolute values, and hence calibration issues and inhomogeneities in the OLR and ERA40 that affect all regions equally. The instrument used to measure the OLR on board the satellite missions is known as the Advanced Very High Resolution Radiometer (AVHRR), a radiometer which measures radiation in four to six channels. While there is a little overlap between these channels and the absorption band for $\mathrm{CO}_{2}$, they do overlap somewhat with other greenhouse gases. The conceptual model presented here, however, does not solely involve $\mathrm{CO}_{2}$, but the net effect of all GHGs, convection, and clouds combined. The estimate of the broad-band spectrum should be independent to that of the line spectra, as the former is a product of Planck emission while the latter is due to absorption of discrete energy quanta due to quantum physics. The OLR measured by the satellites provides mainly an estimate of the broad-band at the top of the atmosphere, however, the estimate is likely contaminated by the line spectra of some of the GHGs in addition to clouds. The thermal emission seen by the satellites is expected to be influenced by both energy flow associated with convection warming the upper levels of the atmosphere as well as that of radiation in various bands. See the supporting material (Supplementary Material) for more details and listings of scripts used for the analysis; it provides both the recipe of the analysis as well as additional figures, and hence, a complete transparency of this analysis through computer code for replication of the results and further experimenting.

\section{Results}

\subsection{The altitude of the emission level}

The $254 \mathrm{~K}$ isotherm $Z_{T 254 K}$ represents the equivalent altitude where earth's bulk heat emission (averaged over all 
altitudes and wavelengths) takes place, and has been an upward trend of $23 \mathrm{~m} / \mathrm{decade}$. This trend is consistent with a deepening of the optical depth, as well as a global mean surface warming of $0.12 \mathrm{~K} /$ decade over the period 1979 2011. It is not widely different to published warming rates of 0.14 to $0.18 \mathrm{~K} /$ decade (Foster and Rahmstorf 2011). Figure 1 shows two different estimates of $Z_{T 254 K}$ based on the ERAINT and the NCEP/NCAR reanalysis (black and grey curves, respectively). There is a good general agreement between the two, albeit with some differences in the details. This means that an observer in space would see IR radiation emanating from shallower atmospheric depths according to Eq. 1 as the GHGs have increased over time (Solomon et al. 2007).

\subsection{Interference between upwelling IR and absorbents}

Figure 2 shows the time evolution of the spatial correlation between the OLR and the $T(2 m)^{4}$ (units in degree Kelvin) from the ERA40 reanalysis. The correlation estimates are in general high due to the warm tropics and cool polar regions since the heat loss in general is highest at lower latitudes.

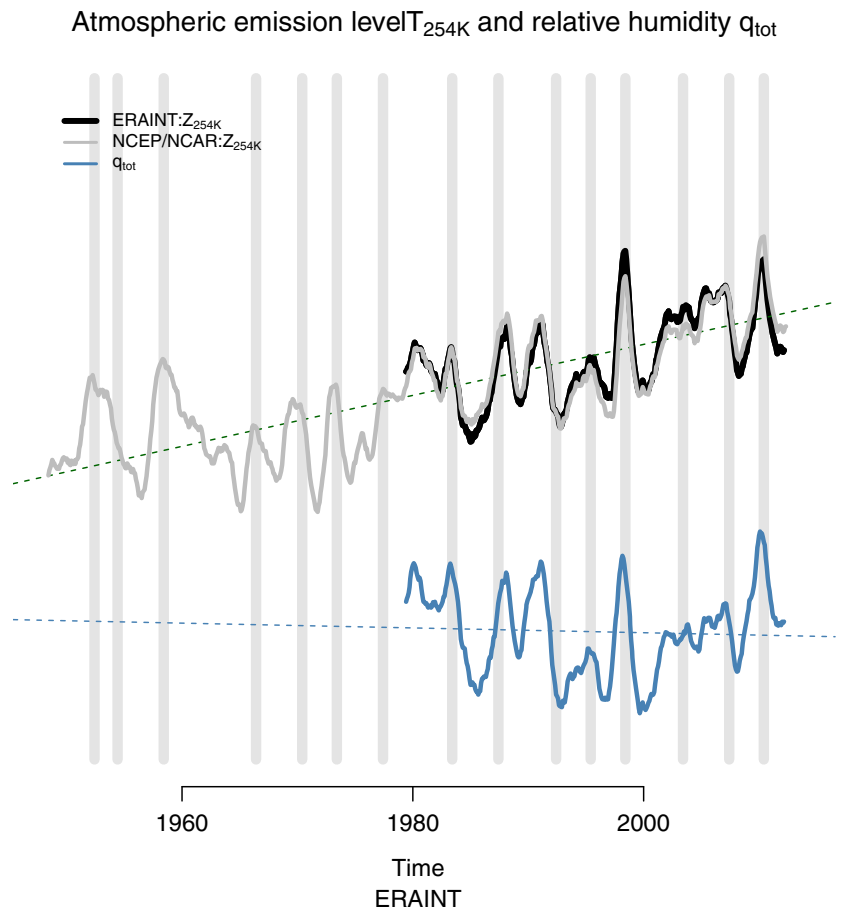

Fig. 1 The 12-month moving mean of the mean global total column water vapor $\left(q_{\mathrm{tot}} ;\right.$ blue $)$, and the bulk emission level altitude $\left(Z_{T 254 K} ;\right.$ black) from ERAINT. The mean $Z_{T 254 K}$ was estimated to be $7219 \pm 2 \mathrm{~m}$, and the trend in the altitude of the bulk emission level, $(23 \pm 2) \mathrm{m} /$ decade, supports the notion of increased optical depth and hence and enhanced GHE. The trend in $Q_{\text {tot }}$ was $(-0.018 \pm$ $0.017) \mathrm{kg} /\left(\mathrm{m}^{2}\right.$ decade $)\left(\right.$ mean $\left.=29 \mathrm{~kg} / \mathrm{m}^{2}\right)$. The curves are plotted here with arbitrary scales along the $y$-axis. The grey vertical bands mark January 1st of known El Niño years

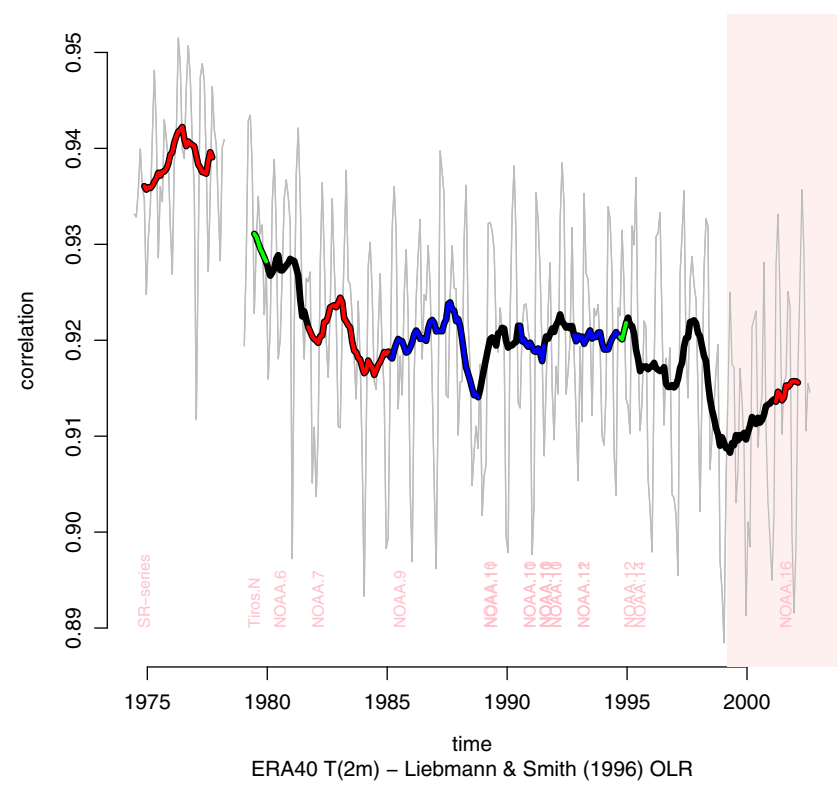

Fig. 2 The spatial correlation between the OLR and $T(2 m)^{4}$ (in degree Kelvin) estimated for each month over the period 1975-2002. The grey curve is unfiltered results and the thick black shows a 12month moving average. The long-term reduction is consistent with a more blurred OLR pattern compared to the surface temperature. The different satellite missions are shown in different colors for the lowpass filtered curve. The faint pink region marks new interpolation scheme

There are also large short-term variations, which are likely to be affected by annual changes in the cloud-cover. Such natural variations are omnipresent on earth, and we do not expect to see a clean straight trend. Nevertheless, the estimates over the interval 1975-2002 suggest a decreasing trend which is consistent with the spatial structure of the OLR becoming more diffuse due to greater opacity, either due to increased GHE or changed cloudiness. High cloud tops, however, are consistent with high $Z_{T 254 K}$, whereas the OLR from low cloud tops are more similar to the upwelling IR from the surface. The clouds are not just a visible aspect of the vertical energy flow, however, they are also a part of the hydrological cycle.

\subsection{Vertical overturning}

Figure 3 shows the overturning activities quantified in terms of the index $\eta_{z}(t)$ (see the figure caption and the Supplementary Material) for three different vertical sections of the troposphere. This overturning index was estimated by taking the global mean of the variance of the area-scaled vertical velocity at a given altitude, and then take the sum over different vertical levels (see Supplementary Material). The variability above $\sim 1000 \mathrm{~m}$ above sea level (a.s.l) has, according to ERAINT, increased since 1995, with most pronounced increase in the middle troposphere $(\sim 1-6.5 \mathrm{~km}$ 
Atmospheric overturning anomaly

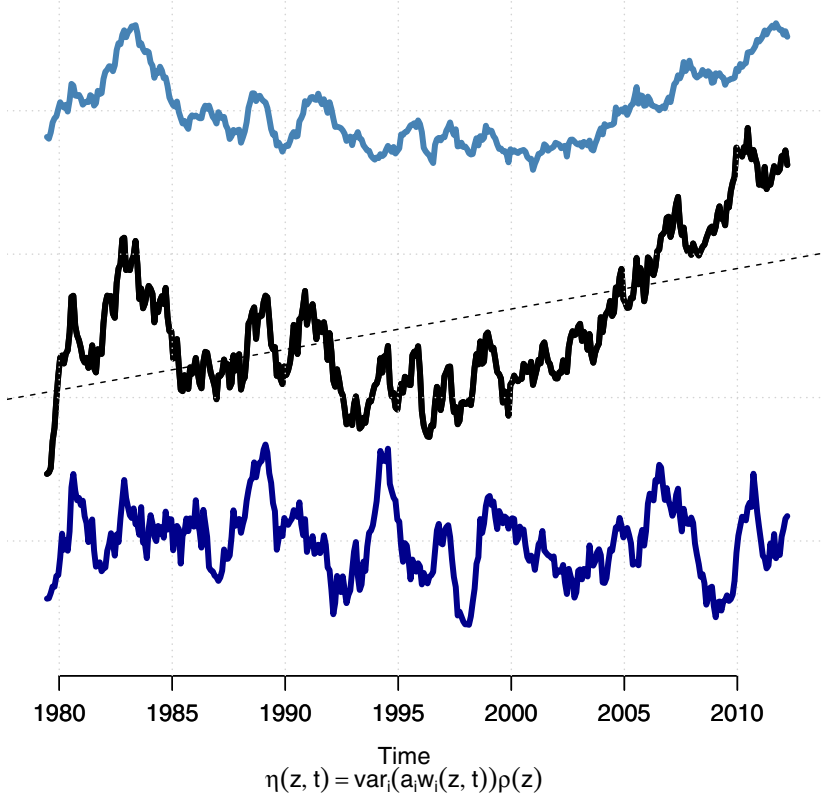

Fig. 3 The 12-month moving mean of the spatial variance of the vertical volume transport (variance of $w$ over space: $\eta_{z}(t)=$ $\operatorname{var}_{i}\left(a_{i} w_{i}(z, t)\right)$ where $i$ is an index over space, $a_{i}$ is the area of gridbox $i$, and $w_{i}(z, t)$ corresponding vertical velocity at time $t$ and altitude $z$ ) from ERAINT. The trend in the atmospheric overturning, $(1100 \pm 90) \mathrm{kg} \mathrm{s}^{-1} /$ decade, in the atmospheric middle levels (black; $1000-6500 \mathrm{~m}$ ) supports the notion of increased optical depth and hence and enhanced GHE. The top pale blue curve shows the upper level $(z>6500 \mathrm{~m})$ and the lower dark blue the surface level $(z<1000 \mathrm{~m})$. The curves are plotted here with arbitrary scales along the $y$-axis

a.s.l.; black). The upward trend in the middle atmosphere is consistent with the notion that increased convection compensates for reduced radiative transfer between the ground and $Z_{T 254 K}$. There is no simple linear trend due to a presence of natural variations, but this interpretation is based on the mean change over the entire record. The upper layer above $\sim 6.5 \mathrm{~km}$ a.s.l. also exhibits a smaller trend, but this layer may have been affected by the convective activity below.

The blue curve in Fig. 1 shows the vertical integral of total column water vapor $\left(q_{\text {tot }}\right)$, exhibiting an insignificant trend. Hence, the increased overturning supports the notion of an enhanced vertical latent heat transport. The excursions in both $q_{\text {tot }}$ and $Z_{T 254 K}$ in 1988, 1991-1992, 1997-1998, and 2009-2010 may be associated with the El Niño Southern Oscillation (ENSO) (Philander 1989) and demonstrate how the optical depth is affected by changes in the atmospheric moisture or the clouds.

Peaks associated with ENSO, seen in the $Z_{T 254 K}$, however, are less visible in the correlation between the OLR and surface temperature, although there may be weak hints of low correlation around some of the El Niño years. One explanation for a weak ENSO signature in the correlation may be that the OLR is expected to be sensitive to the cloud cover, which may dominate on the short time scales and that ENSO involves longitudinal shifts rather than latitudinal changes (See the Supplementary Material).

The global variations in $\eta_{z}(t)$ in the lower $1000 \mathrm{~m}$ have an inter-annual time scale, but exhibit no clear association with ENSO. This layer embeds most of the planetary boundary layer (PBL), which is affected by the surface friction and turbulent mixing, whereas the air above represents the free atmosphere (Schneider and Dickinson 1974). In the lower PBL, the main energy transport takes place through smallscale turbulence with short time scales which may not be well represented by monthly mean values from the atmospheric model used for the reanalysis. Furthermore, the PBL $w$ may not have been assimilated well, due to scarce observations for that level and the incomplete understanding of this lowest layer of air.

\section{Discussion}

The results here suggest that a simple conceptual physics model provides an approximate description of the GHE, by treating all the IR light as one bulk heat loss at the top of the atmosphere where it leaves the planet. It is based on an energy balance, as in Eq. 1, and defines the bulk emission altitude as the region where the temperature equals the emission temperature $T=T_{e}$ (Houghton 1991). This conceptual model does by no means replace more sophisticated lineabsorption or general circulation models, however, the purpose of this conceptual model is to picture the bare essential aspect of the GHE. A similar treatment of the bulk emission and its vertical level is also the basis for the discussion on the 'saturation effect' in the report 'The Copenhagen Diagnosis' (2009)(Allison et al. 2009). Indeed, this conceptual model explains why 'saturation' is not an issue for the GHE, neither on earth nor on Venus. The reason is that an increase in $Z_{T 254 K}$ will lead to a warming at the surface as long as the lapse-rate $\gamma$ is approximately constant. The explanation for why $Z_{T 254 K}$ changes as a response to increasing IR opacity of the atmosphere is that emissions from a constant height will to a greater degree become re-absorbed when the air becomes increasingly opaque at or above this level. It will therefore no longer represent the depth from which the planetary heat loss takes place. A more conventional, but nevertheless physical consistent, view is that the increased $Z_{T 254 K}$ is a consequence of increased surface temperature and a mean lapse rate determined by the atmospheric composition (Santer et al. 2003).

The trend analysis for the $254 \mathrm{~K}$ isotherm $Z_{T 254 K}$ can also be considered as an extension of work by Santer et al. (2003), who reported a robust, zero-order increase 
in tropopause height over 1979-1997 in two earlier versions of reanalyses, which they interpreted as an integrated response to anthropogenic forced warming of the troposphere and cooling of the stratosphere. Here, this aspect is put into a simple physics context of energy flow and Eq. 1, where the metric $Z_{T 254 K}$ is interpreted as an equivalent mean level for the bulk emission. This type of representation is a simplification on par with 'model physics' such as parametrisation of clouds in the GCMs themselves. Models will always be 'flawed' in some sense. Even if one were to include cloud resolving models to describe clouds in GCMs, they too would not represent the cloud micro-physics in detail (Rogers and Yau 1989). Furthermore, models of cloud micro-physics do not fully incorporate the quantum effects and the individual atoms. Moreover, simple models are strictly not correct but may nevertheless be useful; Budyko (1974) observed that 'some aspects of climate genesis might be elucidated by means of the simplest models' (Schneider and Dickinson 1974). An analogy to $Z_{T 254 K}$ is the use of 'virtual images' in optics to compute focal points, and likewise, the emission level diagnostic is not actually an emission level, but is just the height of the temperature that corresponds to the bulk emission level on the right hand side of Eq. 1.

One objection to the interpretations presented here may be that it is difficult to see how OLR measurements are sufficiently accurate to detect the expected $1 \mathrm{~W} / \mathrm{m}^{2}$ change in OLR due to $\mathrm{CO}_{2}$, or that the OLR measurements, which are determined from the AVHRR IR window and crudely converted to an equivalent broadband values, have little sensitivity to most of the spectral absorption associated with $\mathrm{CO}_{2}$. However, this conceptual model does not claim to pick up the net effect of $\mathrm{CO}_{2}$, but describes the aggregated effect of all GHGs as well as all feedback mechanisms such as clouds. Part of the changes in the spatial correlations is expected to be due to convection and clouds, which too play a role for the $\mathrm{GHG}$, in addition to influencing the planetary albedo. Hence, the spatial correlation between the OLR and surface temperature provides an indication of the degree of influence of GHGs on all heat flow from the surface to the top of the atmosphere. Clouds play a role, are a part of the GHE, and are expected to influence $Z_{T 254 K}$. In sum, both the OLR measured by satellite and the energy balance of the planets in our solar system indicate that Planck's law and a bulk emission temperatures provide an approximate description of the planet's temperature (see Supplementary Material).

The objective here was to provide a simple picture of an enhanced GHE and an 'educational toy model' (Schneider and Dickinson 1974) that is consistent with the most up-to-date analysis of the atmosphere. The analysis demonstrates that the numbers support the theory, even though the observations and the reanalyses also have their limitations.
The reanalyses and the OLR provide the to-date best picture that we have of the atmosphere and are an improvement over previous studies (Santer et al. 2003), but there are some caveats as the introduction of new instruments, such as satellite instruments, will result in inhomogeneities (http:// climatedataguide.ucar.edu/) (Hines et al. 2000; Bengtsson et al. 2004). Other issues may concern calibration problems with the OLR, changes in spectral channels, or orbit drifts. However, the analysis was designed to minimize such errors, as the spatial correlation estimates are not sensitive to the absolute value but the changes in the spatial structure.

Another issue may be that the representation of the energy balance may be incomplete merely from the spatial variance of analyzed vertical velocity, as unresolved convective transport (cumulus convection) may be due to updrafts not resolved by reanalysis. While this is true, the atmospheric model used to generate the reanalysis must by-pass this shortcoming by letting other resolved processes provide a means for the unresolved flux of energy, mass, and momentum. Most convective transport must be accounted for by regions of subsidence in the reanalysis in order to be physically consistent.

The OLR-T(2m) correlation is quite steady between 1985 and 1997, and the tropospheric overturning does not have a positive trend between 1980 and 1990. These features may be due to the presence of natural variations, such as ENSO and changes in clouds, but may also be due to a shift in the balance between surface warming and convective fluxes according to the first law of thermodynamics: $c d T_{\mathrm{s}} / d t=$ $S_{0}(1-A) / 4-\left(\sigma T_{s}^{4}+F_{\mathrm{c}}-F_{\mathrm{DLW}}\right)$, where $F_{\mathrm{c}}$ is an energy flux in terms of convective processes and $F_{\mathrm{DLW}}$ the downward radiative flux from the atmosphere. The global mean temperature was subject to a pronounced increase between 1980 and 1998.

One interesting science question is whether a response to changes in the GHG concentration mainly will involve a global mean temperature change $d T_{\mathrm{s}} / d t$, or if the response leads to changes in the hydrological cycle turn-around rate $\eta_{\mathrm{Z}}(t)$. This question has some relevance to the recent conundrum regarding the so-called 'hiatus' (Kosaka and Xie 2013). It is interesting to note the clear upward change in the mid-level tropospheric overturning during this period. The more conventional pure reductionist radiative model approach (Sloan and Wolfendale 2013; Manabe and Möller 1961 ) is unable to explain a lack of warming in the presence of increased GHGs unless additional factors are included such as natural variations (Easterling and Wehner 2009). Natural variations, however, may be explained in the proposed conceptual radiative-convection model as changes in the partitioning between radiative and convective energy flows $F_{\mathrm{DLW}}$ and $F_{\mathrm{c}}$. Furthermore, $Z_{T 254 K}$ does not show any slow-down over the recent years despite the 'hiatus' (Fig. 1), but is more consistent with the steady increase in 
the global mean sea level (IPCC synthesis report A 2014). There are also indications that the 'hiatus' is related to increased warming in the deep oceans (Balmaseda et al. 2013) and that the estimate of the global mean has been underestimated due under-reporting of the recent warming in the Arctic (Cowtan and Way 2014).

The consideration of the vertical energy flow, where latent heat transfer and convective processes compensate for reduced IR energy transfer, entangles the GHE with the hydrological cycle. Radiation-transfer considerations alone do not provide a complete picture of the real situation connected to the AGW, as convection and latent heat both set the atmosphere's vertical temperature profile and involve evaporation, condensation, and precipitation. From these principles, an increased GHE is expected to change rainfall patterns (Benestad 2013). Furthermore, this framework embeds more sophisticated explanations than traditional purely radiative models and incorporates effects from feedback processes through $d T / d z$, response in $Z_{T 254 K}$, and the albedo $A$. The albedo was assumed to be a constant here for simplicity, but it could potentially vary over time due to changed cloudiness, vegetation, and snow/ice. Estimated variations in the albedo has been marginal (of the order of $2 \%$ ) over the last decades (Pallé et al. 2004), however, the conceptual model could account for such variations if $A$ is replaced by satellite-borne observations of short-wave radiation reflected by the earth $A(t)$. A $2 \%$ increase in the reflectance anomaly is roughly equivalent to $6 \mathrm{~W} / \mathrm{m}^{2}$ in terms of short wave radiative forcing, but nevertheless small compared to the 15-20\% seasonal variability in earth's albedo (Pallé et al. 2004).

Although the conceptual model presented here was similar to the ideas discussed by Hulburt in 1931, he did not emphasise the vertical energy flow, the hydrological cycle, and changes in the greenhouse gas concentrations. These aspects were implicit in his conceptual model; however, he did not extend these to the question of climate change and the entanglement between the hydrological cycle and the GHE. An interesting question is whether reviving this old physics-model in the context of modern data can convince the scientifically literate who still do not acknowledge an AGW. This conceptual model also explains why some contrarian views based on IR flux are flawed (Monckton et al. 2015): They do not account for convection. There is a cause for optimism if the reason for different views is 'agnotological' in terms of forgotten ideas (Proctor and Schiebinger 2008). This toy model is probably too complex for the average person who is not in a scientific (or analytic) field and will probably not be enough to make the society grasp the challenges connected with an increased greenhouse effect, but it may be one incremental step towards an enhanced general understanding. The conceptual model can be followed up with an explanation for why other views are wrong through replication and falsification (Benestad et al. 2015). Moreover, a broader scientific consensus may make a stronger impression by showing how science works in terms of finding the most credible explanation.

\section{Conclusions}

The GHE is a result of a vertical distance between a planet's surface, where energy from the sun is deposited, and higher aloft, where its heat loss back to space takes place. The vertical distance itself, however, is determined by GHGs. This picture is derived from well-understood and elementary physics, and the validation shows that this conceptual model is consistent with the best data there is. It provides non-traditional diagnostics of an increased GHE that are consistent with the most up-to-date reanalyses and OLR. The reanalyses themselves may involve serious caveats in terms of time-dependent errors and biases, and the analyses cannot ascertained whether these trends are real. The long-term trends identified in the reanalyses and the OLR measurements are nevertheless consistent with the notion of increasing opacity for IR light, elevation of the OLR emission level, and convective activity. The simple conceptual model also provides a link between the GHE and the hydrological cycle and offers explanations for some issues that have been up for popular public debate, showing why the issue of 'saturation' is not relevant and why a 'hiatus' is not in violation with an increased greenhouse effect.

Acknowledgments This work has been partly supported by the Norwegian Meteorological Institute as a means of enlightening the general public on climate change. Part of this work is based on two posts on RealClimate.org in 2010, and I acknowledge valuable discussions in the threads of these posts and am grateful to Ketil Isaksen for useful comments on the manuscript. The analysis was also based on the effort done by the European Centre for Medium-range Weather Forecasts (ECMWF) in terms of producing the ERAINT reanalysis. The ERAINT and ERA40 reanalyses can be obtained from http://www. ecmwf.int (monthly); the NCEP/NCAR reanalyses from http://www. esrl.noaa.gov/psd/data/gridded/data.ncep.reanalysis.html. Interpolated OLR data provided by the NOAA/OAR/ESRL PSD, Boulder, Colorado, USA, from their Web site at http://www.esrl.noaa.gov/psd/. The processed data is also available from the open-source R-package (Benestad 2014a).

Open Access This article is distributed under the terms of the Creative Commons Attribution 4.0 International License (http:// creativecommons.org/licenses/by/4.0/), which permits unrestricted use, distribution, and reproduction in any medium, provided you give appropriate credit to the original author(s) and the source, provide a link to the Creative Commons license, and indicate if changes were made. 


\section{References}

Abraham J, Cook J, Fasullo J, Jacobs P, Mandia S, Nuccitelli D (2014) Review of the consensus and asymmetric quality of research on human-induced climate change. Cosmopolis (1)

Allison I, Bindoff N, Bindschadler R, Cox P, de Noblet N, England M, Francis J, Gruber N, Haywood A, Karoly D, Kaser G, Quéré CL, Lenton T, Mann M, McNeil B, Pitman A, Rahmstorf S, Rignot E, Schellnhuber H, Schneider S, Sherwood S, Somerville R, Steffen K, Steig E, Visbeck M, Weaver A (2009) The copenhagen diagnosis, 2009: Updating the world on the latest climate science. Tech. rep. The University of New South Wales Climate Change Research Centre (CCRC), Sydney

Anderegg W, Prall J, Harold J, Schneider S (2010) Expert credibility in climate change. PNAS 107(27):12,107-12,110. doi:0.1073/pnas.1003187107

Arrhenius S (1896) On the influence of carbonic acid in the air upon the temperature of the ground. Philosophical Magazine and Journal of Science 41:237-276

Balmaseda M, Trenberth K, Källén E (2013) Distinctive climate signals in reanalysis of global ocean heat content. Geophys Res Lett 40:1754-1759. doi:10.1002/grl.50382

Benestad R (2014a) Ghe. Tech. rep., figshare. doi:10.6084/m9.fig share. 1141971

Benestad R (2014b) IPCC: a failure in communicating the impact of new findings. Earthmagazine. http://www.earthmagazine.com/ article/ipcc-failure-communicating-impact-new-findings

Benestad RE, Nuccitelli D, Lewandowsky S, Hayhoe K, Hygen HO, van Dorland R, Cook J (2015) Learning from mistakes in climate research. Theor Appl Climatol. doi:10.1007/s00704-015-1597-5

Benestad RE (2013) Association between trends in daily rainfall percentiles and the global mean temperature. J Geophys Res-Atmos 118(19):10,802-10,810. doi:10.1002/jgrd.50814

Bengtsson L, Hagemann S, Hodges K (2004) Can climate trends be calculated from reanalysis data? J Geophys Res 109. doi:10.1029/2004JD004536

Boykoff M (2008) Lost in translation? united states television news coverage of anthropogenic climate change, 1995-2004. Clim Chang 86:1-11

Charney J, Arakawa A, Baker D, Bolin B, Dickinson R, Goody R, Leith C, Stommel H, wunsch C (1979) Ad hoc study group on carbon dioxide and climate. Ad hoc study group, Climate Research Board, National Research Council, USA

Cook J, Nuccitelli D, Green S, Richardson M, Winkler B, Painting R (2013) Quantifying the consensus on anthropogenic global warming in the scientific literature. Environ Res Lett 8(024024)

Cowtan K, Way RG (2014) Coverage bias in the hadcrut4 temperature series and its impact on recent temperature trends. Q J R Meteorol Soc 140(683)

Doran PT, Zimmerman MK (2011) Examining the scientific consensus on climate change. Eos (90):22-23

Easterling D, Wehner M (2009) Is the climate warming or cooling? Geophys Res Lett 36:L08,706. doi:10.1029/2009GL037810

Fleagle R, Businger J (1980) An Introduction to Atmospheric Physics, International Geophysics Series, vol 25, 2nd edn. Academic Press, Orlando

Foster G, Rahmstorf S (2011) Global temperature evolution 19792010. Environ Res Lett 6:044,022. doi:10.1088/1748-9326/6/4/044022

Gerlich G, Tscheuschner RD (2009) Falsification of the atmospheric $\mathrm{CO}_{2} \$$ greenhouse effects within the frame of physics. Int J Mod Phys B 23(03):275364. doi:10.1142/S021797920904984X

Halpern JB, Colose CM, Ho-Stuart C, Shore JD, Smith AP, Zimmermann J (2010) Comment on falsification of the atmospheric CO
${ }_{2} \$$ greenhouse effects within the frame of physics. Int J Mod Phys B 24(10):13091332. doi:10.1142/S021797921005555X

Hartmann D (1994) Global Physical Climatology. Academic Press, San Diego

Hernes G (2012) Hot Topic - Cold Comfort, Climate Change and Attitude Change. ISBN 978-82-93089-03-2, NordForsk, Oslo, Norway

Hines K, Bromwich D, Marshall G (2000) Artificial surface pressure trends in the ncep-ncar reanalysis over the southern ocean and antarctica. J Climate 13:3940-3952

Houghton J (1991) The physics of atmospheres. Cambridge University Press, Cambridge

Hulburt EO (1931) The temperature of the lower atmosphere of the earth. Phys Rev 38:1876-1890. doi:10.1103/PhysRev.38.1876

IPCC synthesis report A (2014) Climate change 2014 (longer report). Tech. rep., Intergovernmental Panel on Climate Change, http://ipcc.ch/pdf/assessment-report/ar5/syr/SYR_AR5_ LONGERREPORT.pdf

Kalnay E, Kanamitsu M, Kistler R, Collins W, Deaven D, Gandin L, Iredell M, Saha S, White G, Wollen J, Zhu Y, Chelliah M, Ebisuzaki W, Higgins W, Janowiak J, Mo K, Ropelewski C, Wang J, Leetmaa A, Reynolds R, Jenne R, Joseph D (1996) The NCEP/NCAR 40-year reanalysis project. Bull Amer Meteor Soc $77: 437-471$

Keeling C, Bacastow R, Bainbridge AE, JR CE, Guenther P, Waterman L, Chin J (1976) Atmospheric carbon dioxide variations at mauna loa observatory, hawaii. Tellus 6(XXVIII):538551

Kopp G, Lean JL (2011) A new, lower value of total solar irradiance: evidence and climate significance. Geophys Res Lett 38:L01706. doi:10.1029/2010GL045777

Kosaka Y, Xie SP (2013) Recent global-warming hiatus tied to equatorial pacific surface cooling. Nature 501(7467):403-407. doi:10.1038/nature 12534

Lacis A, Schmidt G, Rind D, Ruedy R (2010) Atmospheric co2: principal control knob governing earths temperature. Science 330:353-359. doi:10.1126/science. 1190653

Leviston Z, Walker I, Morwinski S (2012) Your opinion on climate change might not be as common as you think. Nat Clim Change (3):334-337

Lewandowsky S, Gignac GE, Oberauer K (2013) The role of conspiracist ideation and worldviews in predicting rejection of science. PloS One 8(e75637)

Liebmann S (1996) Description of a complete (interpolated) outgoing longwave radiation dataset. Bull Amer Meteor Soc 77(June): $1275-1277$

Manabe S, Möller F (1961) On the radiative equilibrium and heat balance of the atmosphere. Mon Weather Rev 89(12):503532

Manabe and Strickler (1964) Thermal equilibrium of the atmosphere with a convective adjustment journal of the atmospheric sciences, $21: 361-385$

McCuin JL, Hayhoe K, Hayhoe D (2014) Comparing the effects of traditional vs. misconceptions-based instruction on student understanding of the greenhouse effect. J Geol Educ: August 2014 62(3):445-459

Monckton C, Soon WW-H, Legates DR, Briggs WM (2015) Why models run hot: results from an irreducibly simple climate model. Sci Bull 60(1):122-135

North G (1975) Theory of energy-balance climate models. J Atmos Sci 32(11):2033-2043

Oreskes N (2004) The scientific consensus on climate change. Science 306(5702):1686. doi:10.1126/science.1103618 
Oreskes N, Conway E (2008) Agnotology: The Making and Unmaking of Ignorance. In: Proctor R, Schiebinger L (eds) chap Challenging Knowledge. Stanford University Press. ISBN: 978080475 9014

Pallé E, Goode PR, Montañés-Rodriguez P, Koonin SE (2004) Changes in earth's reflectance over the past two decades. Science 304(5675):1299-1301. doi:10.1126/science.1094070

Peixoto J, Oort A (1992) Physics of Climate.AIP, N.Y

Philander S (1989) El Niño, La Niña, and the Southern Oscillation. Academic Press, N.Y

Pierrehumbert R (2011) Infrared radiation and planetary temperature. Physics Today (64):33-38

Proctor R, Schiebinger L (2008) Agnotology: The Making and Unmaking of Ignorance. Stanford University Press. ISBN: 9780804759014

Rahmstorf S (2012) Is journalism failing on climate Environ Res Lett 7(041003)

Rogers R, Yau M (1989) A Short Course in Cloud Physics, 3rd edn. Pergamon Press, Oxford

Santer B, Sausen R, Wigley T, Boyle J, AchutaRao K, Doutriaux C, Hansen J, Meehl G, Roeckner E, Ruedy R, Schmidt G, Taylor KE (2003) Behavior of tropopause height and atmospheric temperature in models, reanalyses, and observations: Decadal changes. J Geophys Res 108:4002. doi:10.1029/2002JD002258

Schneider S, Dickinson R (1974) Climate modeling. Rev Geophys 12(3):447-493. doi:10.1029/RG012i003p00447

Simmons A, Gibson J (2000) The era-40 project plan. ERA-40 Project Report Series 1, ECMWF, www.ecmwf.int
Simmons A, Uppala S, Dee D, Kobayashi S (2007) Era-interim: New ecmwf reanalysis products from 1989 onwards. ECMWF Newsletter

Sloan T, Wolfendale A (2013) Cosmic rays, solar activity and the climate. Environ Res Lett 8:045,022. doi:10.1088/1748-9326/8/4/ 045022

Smith AP (2008) Proof of the atmospheric greenhouse effect. arXiv:08024324

Solomon S, Quin D, Manning M, Chen Z, Marquis M, Averyt K, Tignotr M, Miller H (2007) Climate Change: The Physical Science Basis. Contribution of Working Group I to the Fourth Assessment Report of the Intergovernmental Panel on Climate Change. Cambridge University Press, United Kingdom andNew York

Stocker T, Qin D, Tignor GKPM, Allen S, Boschung J, Nauels A, Xia Y, Bex V, Midgley P (2013). Cambridge University Press, Cambridge, United Kingdom and New York

Theissen KM (2011) What do u.s. students know about climate change? Eos (92):477-478

Trenberth K (2011) Changes in precipitation with climate change. Clin Res 47:123-138

Trenberth K, Stepaniak DP (2004) The flow of energy through the earths climate system. Q J R Meteorol Soc 130:26772701. doi:10.1256/qj.04.83

Wang WC, Stone P (1980) Effect of ice-albedo feedback on global sensitivity in a one-dimensional radiative-convective climate model. J Atmos Sci 37:545-552. doi:10.1175/1520-0469(1980)037

Weart S (2003) Discovery of Global Warming. Harvard University Press 